\title{
Cross-border emergence of clonal lineages of ST38 Escherichia coli producing the OXA-48-like carbapenemase OXA-244 in Germany and Switzerland
}

\author{
Linda Falgenhauer ${ }^{\mathrm{a}, \mathrm{b}}$, Patrice Nordmann ${ }^{\mathrm{c}, \mathrm{d}, \mathrm{e}}$, Can Imirzalioglu ${ }^{\mathrm{a}}$, Yancheng Yao ${ }^{\mathrm{a}}$, \\ Jane Falgenhauer ${ }^{\mathrm{a}}$, Anja M. Hauri ${ }^{\mathrm{f}}$, Petra Heinmüller ${ }^{\mathrm{f}}$, Trinad Chakraborty ${ }^{\mathrm{a}, *}$ \\ ${ }^{a}$ Institute of Medical Microbiology, and German Center for Infection Research (DZIF), Partner Site Giessen-Marburg-Langen, Justus Liebig University Giessen, \\ Giessen, Germany \\ ${ }^{\mathrm{b}}$ Institute of Hygiene and Environmental Medicine, Justus Liebig University Giessen, Giessen, Germany \\ ${ }^{\mathrm{c}}$ Medical and Molecular Microbiology, Section of Medicine, Faculty of Science and Medicine, University of Fribourg, Fribourg, Switzerland \\ d INSERM European Unit (IAME, France), University of Fribourg, Fribourg, Switzerland \\ e Swiss National Reference Center for Emerging Antibiotic Resistance (NARA), University of Fribourg, Fribourg, Switzerland \\ ${ }_{\mathrm{f}}^{\mathrm{f}}$ Department of Epidemiology, Hesse Health Office, Dillenburg, Germany
}

\begin{abstract}
Background: Carbapenemase-producing Gram-negative bacteria cause infections that are difficult to treat and represent a rising threat to healthcare systems worldwide. This study analysed isolates of Escherichia coli (E. coli), a species associated with nosocomial-acquired and community-acquired infections, from hospitals in Germany and Switzerland exhibiting a slight decrease in susceptibility to carbapenems.

Methods: E. coli strains from Germany and Switzerland, obtained mainly in 2019, were first screened for carbapenemase genes by PCR and subsequently whole-genome-sequenced and analysed for their clonal relationship using multilocus sequence typing, single nucleotide polymorphisms, virulence and antibioticresistance gene content.

Results: The analysis revealed the presence of extended $\beta$-lactamase (ESBL)-producing $E$. coli clones producing OXA-244, a point-mutation derivative of OXA-48, with a predominance of isolates exhibiting the sequence type (ST) ST38 in both Germany and Switzerland. These clustered exclusively into two distinct lineages: one encoding CTX-M-27, a recently emerged extended-spectrum $\beta$-lactamase, and the other CTX-M-14b. All OXA244/CTX-M-27 ST38 isolates harboured the Dr adhesin operon and a representative isolate exhibited a diffuse adherence (DAEC) phenotype and was invasive for Hela cells.

Conclusion: Clonal lineages of ST38 are members of $E$. coli phylogenetic group D commonly associated with extra-intestinal infections. Their increased isolation in two different European countries indicates ongoing spread of ST38 ESBL-producing and OXA-244-producing E. coli clonal lineages. It is possible that members of the multidrug-resistant DEAC ExPEC group have expanded globally, but that this is currently underreported because of the inherent difficulty in detecting isolates expressing the OXA-244 allele.
\end{abstract}

\section{Introduction}

Carbapenem-producing Enterobacterales (CPE) are increasingly being identified not only in Klebsiella pneumoniae but also in Escherichia coli (E. coli) [1]. For E. coli, NDM- and OXA-48-type enzymes are the main determinants of carbapenem resistance [1].

* Corresponding author: Institute of Medical Microbiology, and German Center for Infection Research (DZIF), Partner Site Giessen-Marburg-Langen, Justus Liebig University Giessen, Schubertstrasse 81, 35392 Giessen, Germany. Tel.: +49 64199 41250

E-mail address: trinad.chakraborty@mikrobio.med.uni-giessen.de

(T.
Their presence in E. coli, which is a source of both nosocomial and community-acquired infections, raises the threat of their silent and impossible-to-control spread outside the hospital environment. This study analysed OXA-244-producing E. coli isolates collected in two different European countries.

\section{Materials and Methods}

\subsection{Strain collection}

OXA-244-producing E. coli isolates obtained from two CPE surveillance networks, one in the state of Hesse, Germany 
(ca. 6.2 million inhabitants, SurvCARE http://www.mre-netzwerkmittelhessen.de/material/SurvCARE), and the other in Switzerland (ca. 8.4 millions inhabitants, National Reference Center for Emerging Antibiotic Resistance, NARA) were analysed.

For SurvCARE, extended-spectrum $\beta$-lactam (ESBL)-resistant and carbapenem-resistant isolates were identified using chromogenic media by the laboratories of the participating hospitals. Determination of the MIC towards carbapenems (ertapenem, imipenem and meropenem) was performed using Etest following EUCAST recommendations [2]. Resistance phenotypes of other antibiotics and species were determined with the VITEK $2{ }^{\circledR}$ system (bioMerieux, Nürtingen, Germany). Enterobacterial strains that displayed a decreased susceptibility to carbapenems were regularly sent to either site, where the molecular mechanisms of resistance were analysed [3].

\subsection{Whole genome sequencing and analysis}

For whole genome sequencing, DNA was isolated from overnight cultures using the PureLink Genomic DNA kit (Invitrogen, ThermoFischer, Germany). Short read sequencing libraries were prepared for all E. coli ST-38/OXA-244 isolates ( $\mathrm{n}=31$ ) using the Nextera XT kit (Illumina, Netherlands) and sequenced on MiSeq/NextSeq sequencing machines (read length either $2 \times 300$ nt or $2 \times 150 n t)$. A long-read sequencing library of the isolate SurvCARE133 was prepared using the native barcoding kit (EXP-NBD103, Oxford Nanopore Technologies) and 1D chemistry (SQK-LSK108, Oxford Nanopore Technologies, UK). Sequencing was performed on a MinION sequencer (Oxford Nanopore Technologies, UK) using a SpotON Mk I R9 Version flow Cell (FLO-MIN106, Oxford Nanopore Technologies, UK). Postsequencing quality control, assembly and virulence gene determination was performed using $\mathrm{ASA}^{3} \mathrm{P}$ pipeline [4]. Antibiotic resis- tance genes, serotypes, plasmid incompatibility groups and pMLST types were determined using the Center for Epidemiology tools (http://www.genomicepidemiology.org/). Phylogenetic analysis was performed using HarvestSuite [5]. Regions exhibiting recombinational hotspots were removed using Gubbins [6]. The raw sequencing data information was stored in the National Center for Biotechnology Information under the project number PRJNA602666 (https://www.ncbi.nlm.nih.gov/).

\subsection{Adherence assays}

Human HeLa cervical epithelial cells (ATCC CCL2) were grown and assayed at $37^{\circ} \mathrm{C}$ with $5 \% \mathrm{CO}_{2}$ in Dulbeccos Minimal Essential Medium (DMEM) supplemented with $0.5 \%$ (vol/vol) foetal calf serum (FCS). Fresh bacterial cultures grown overnight in LB medium were used to prepare suspensions in PBS adjusted to an OD of 0.1 at $600 \mathrm{~nm}\left(\sim 1 \times 10^{6} \mathrm{cfu} / \mathrm{mL}\right)$. Subconfluent $10 \%$ buffered formalin-fixed or live 48 -h of HeLa cells grown on coverslips placed in $3.5-\mathrm{cm}$ petri dishes were washed three times (5 minutes each) with PBS and overlaid with bacterial suspensions. Adhesion was allowed to take place for 3 hours at $37^{\circ} \mathrm{C}$ in a $\mathrm{CO}_{2}$ incubator. After incubation, bacterial suspensions were removed, and the monolayers were washed three times in PBS. Live monolayers were fixed with cold methanol for 5 minutes, then both types of monolayers were air-dried and stained with Giemsa diluted 1:20 for 1 hour to visualise bacteria attached to HeLa cells.

\subsection{Bacterial internalisation assay}

Bacterial suspensions in PBS, prepared as described for the adherence assay, were added to monolayers of HeLa cells ( 10 $000 /$ well) to give a multiplicity of infection (MOI) of 10-15 in 24 well plates and incubated for 3 hours at $37^{\circ} \mathrm{C}$ in the $\mathrm{CO}_{2}$ incubator.

Table 1

Clinical data of the Escherichia coli ST38 OXA-244-producing isolates. Origin: CH, Switzerland, GE, Germany.

\begin{tabular}{|c|c|c|c|c|c|c|c|}
\hline Isolate & Year of isolation & Clinic & Origin & Sample type & $\begin{array}{l}\text { Age (years, if not } \\
\text { otherwise stated) }\end{array}$ & Sex & $\begin{array}{l}\text { Infection (I) / } \\
\text { Colonisation (C) }\end{array}$ \\
\hline N11 & 2017 & $\mathrm{CH}-04$ & $\mathrm{CH}$ & Liquid drain & 78 & $\mathrm{M}$ & I \\
\hline N293 & 2018 & $\mathrm{CH}-03$ & $\mathrm{CH}$ & Urine & 67 & $\mathrm{~F}$ & I \\
\hline N460 & 2019 & CH-04 & $\mathrm{CH}$ & Urine & 77 & $\mathrm{~F}$ & I \\
\hline N481 & 2019 & $\mathrm{CH}-05$ & $\mathrm{CH}$ & Urine & 75 & M & I \\
\hline N511 & 2019 & $\mathrm{CH}-02$ & $\mathrm{CH}$ & Rectal swab & 64 & $\mathrm{~F}$ & $\mathrm{C}$ \\
\hline N658 & 2019 & $\mathrm{CH}-05$ & $\mathrm{CH}$ & Urine & 48 & $\mathrm{~F}$ & I \\
\hline N816 & 2019 & $\mathrm{CH}-04$ & $\mathrm{CH}$ & Urine & 41 & $\mathrm{~F}$ & I \\
\hline N832 & 2019 & $\mathrm{CH}-05$ & $\mathrm{CH}$ & Urine & 28 & $\mathrm{~F}$ & I \\
\hline N861 & 2019 & $\mathrm{CH}-01$ & $\mathrm{CH}$ & Rectal swab & 44 & $\mathrm{~F}$ & $\mathrm{C}$ \\
\hline N871 & 2019 & $\mathrm{CH}-06$ & $\mathrm{CH}$ & Urine & 24 & $\mathrm{~F}$ & I \\
\hline N888 & 2019 & $\mathrm{CH}-07$ & $\mathrm{CH}$ & Urine & 62 & $\mathrm{~F}$ & I \\
\hline N930 & 2019 & $\mathrm{CH}-08$ & $\mathrm{CH}$ & Urine & 14 & $\mathrm{~F}$ & I \\
\hline sc38-1 & 2018 & GE-08 & GE & Rectal swab & 37 & $\mathrm{~F}$ & $\mathrm{C}$ \\
\hline SurvCARE133 & 2018 & GE-02 & GE & Rectal swab & 69 & $\mathrm{M}$ & $\mathrm{C}$ \\
\hline SurvCARE134 & 2018 & GE-02 & GE & Rectal swab & 69 & M & $\mathrm{C}$ \\
\hline SurvCARE140 & 2018 & GE-07 & GE & Punctate lower abdomen & 53 & $\mathrm{~F}$ & I \\
\hline SurvCARE303 & 2019 & GE-06 & GE & Rectal swab & 80 & M & $\mathrm{C}$ \\
\hline SurvCARE318 & 2019 & GE-03 & GE & Urine & 75 & $\mathrm{~F}$ & $\mathrm{C}$ \\
\hline SurvCARE319 & 2019 & GE-01 & GE & Rectal swab & 63 & $\mathrm{~F}$ & $\mathrm{C}$ \\
\hline SurvCARE335 & 2019 & GE-10 & GE & Gall bladder secretion & 84 & M & $\mathrm{C}$ \\
\hline SurvCARE377 & 2019 & GE-09 & GE & Rectal swab & 30 & M & $\mathrm{C}$ \\
\hline SurvCARE378 & 2019 & GE-09 & GE & Urine & 30 & $\mathrm{~F}$ & I \\
\hline SurvCARE382 & 2019 & GE-05 & GE & Vaginal swab & 29 & $\mathrm{~F}$ & $\mathrm{C}$ \\
\hline SurvCARE383 & 2019 & GE-05 & GE & Nose/throat swab & 1 month & $\mathrm{F}$ & $\mathrm{C}$ \\
\hline SurvCARE384 & 2019 & GE-05 & GE & Nose/throat swab & 1 month & $\mathrm{F}$ & $\mathrm{C}$ \\
\hline SurvCARE389 & 2019 & GE-05 & GE & Rectal swab & 1 month & $\mathrm{F}$ & $\mathrm{C}$ \\
\hline SurvCARE390 & 2019 & GE-05 & GE & Rectal swab & 1 month & $\mathrm{F}$ & $\mathrm{C}$ \\
\hline SurvCARE392 & 2019 & GE-01 & GE & Rectal swab & 23 & $\mathrm{~F}$ & $\mathrm{C}$ \\
\hline SurvCARE393 & 2019 & GE-01 & GE & Nose/throat swab & 1 month & $\mathrm{F}$ & $\mathrm{C}$ \\
\hline SurvCARE421 & 2019 & GE-05 & GE & Rectal swab & 1 month & $\mathrm{F}$ & $\mathrm{C}$ \\
\hline SurvCARE438 & 2019 & GE-04 & GE & Rectal swab & 4 & $\mathrm{M}$ & $\mathrm{C}$ \\
\hline
\end{tabular}


Cells were subsequently washed thrice with Hanks medium, followed by incubation for 1 hour with DMEM and 0.5\% FCS containing $200 \mu \mathrm{l} / \mathrm{mL}$ gentamycin to kill extracellular bacteria. Cells were washed thrice with Hanks buffer and subsequently lysed by the addition of sterile water containing $0.1 \%$ Triton X-100 for $30 \mathrm{~min}$ utes at room temperature. Appropriate dilutions were plated after the third washing with Hanks buffer and after lysis to determine the number of viable bacteria. Each assay was run in triplicate.

\section{Results and Discussion}

Carbapenemase-producing $E$. coli isolates obtained between 2017 and 2019 from the two CPE surveillance networks SurvCARE (Federal State of Hesse, Germany; $\mathrm{n}=107$ ) and NARA (Switzerland, $\mathrm{n}=448$ ) were analysed for the underlying resistance mechanisms. In both networks, the emergence of OXA-244-producing E. coli isolates among carbapenemase-producing $E$. coli isolates was noted in 2018 and 2019 (Germany 2018: 22.2\%, 2019: 27.5\%; Switzerland: 2018: 1\%, 2019: 7\%) as compared with 2017 (Germany: 0.0\%, Switzerland: $0.0 \%$ ). The enzyme OXA-244 is a point-mutant derivative of OXA-48 (Arg212Gly substitution) with weaker carbapenemase activity [3].
Forty-one E. coli isolates harbouring an OXA-244 carbapenemase were detected and whole genome sequenced to determine their epidemiological relatedness. Using multilocus sequence typing, 31 of the 41 OXA-244 producers (75.6\%, Supplementary Figure 1) clustered as a single E. coli sequence type (ST) 38. Other STs identified included ST69 ( $\mathrm{n}=3)$, ST10 $(\mathrm{n}=1)$, ST58 $(\mathrm{n}=1)$, ST361 $(\mathrm{n}=1)$, ST648 $(\mathrm{n}=1)$, ST963 $(\mathrm{n}=1)$, ST131 $(\mathrm{n}=1)$ and ST1722 $(\mathrm{n}=1)$. The predominance of $E$. coli ST38-OXA-244 producers in both countries suggested a possible ongoing outbreak associated with ST38 isolates.

A total of 31 ST38 OXA-244 isolates from both countries were studied (Table 1, Supplementary Table 1). Nineteen E. coli isolates were obtained from ten hospitals in Hesse and 12 isolates derived from 12 hospitals/private clinics in Switzerland. Isolates were obtained from urine samples $(n=10)$, rectal swabs $(n=15)$, vaginal smear/swab $(n=2)$ and other sources $(n=4)$. Isolates originated from different parts of Switzerland (German-, French- and Italianspeaking regions) and in the state of Hesse (Germany) from hospitals distributed within an area half the size of Switzerland. E. coli ST38 are representatives of the E. coli phylogenetic group D, commonly associated with extra-intestinal infections (ExPEC) [7].

Detailed analysis indicated the presence of two major clonal lineages that segregated almost entirely along the extended-spectrum

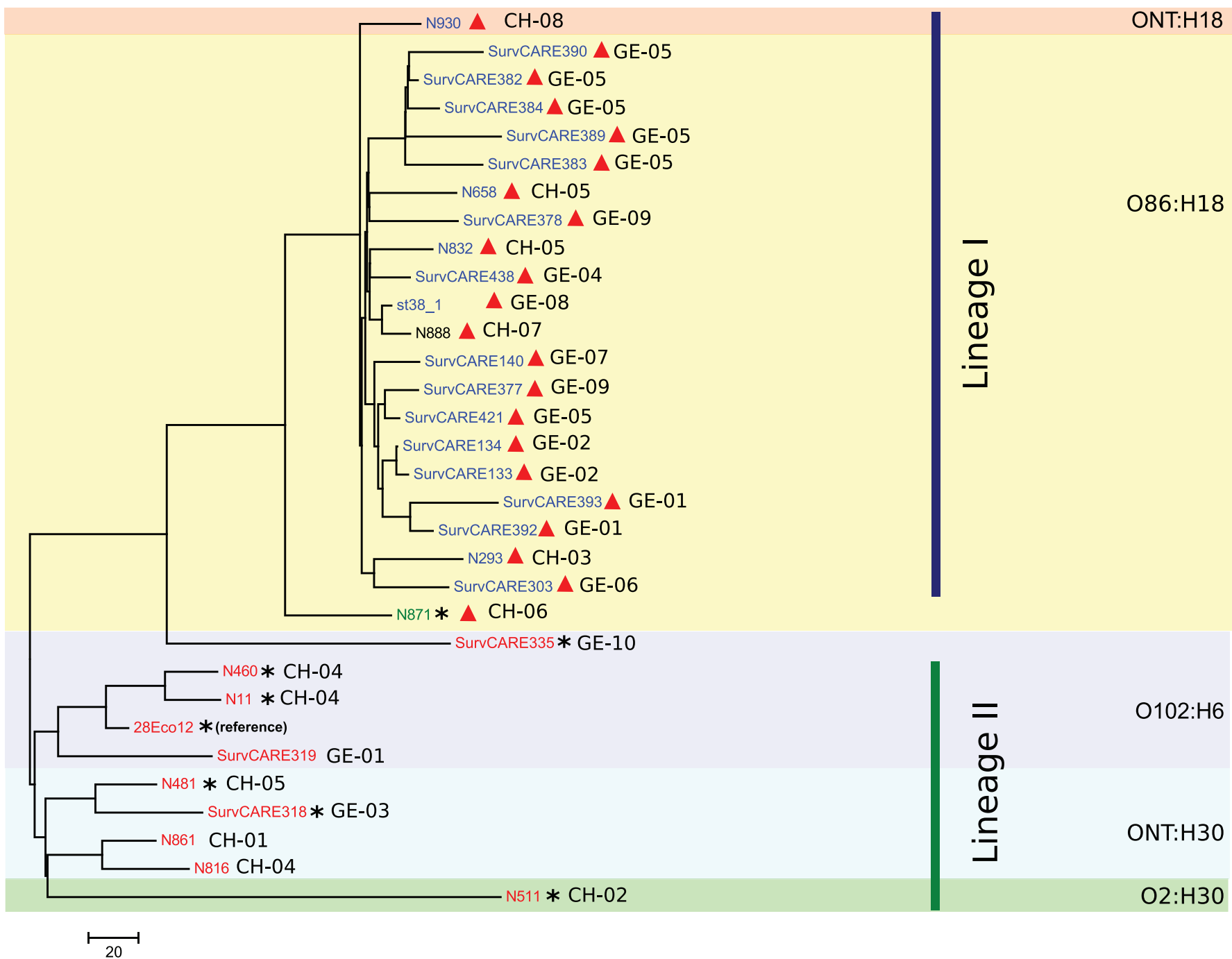

Figure 1. Core-genome-based analysis of OXA-244 producing ST38 isolates. The colour of the isolate names indicates the carriage of additional ESBL/AmpC enzymes; CTXM-14b, red, CTX-M-27, blue, CMY-2, green; no additional ESBL/AmpC gene, black. A star indicates the presence of a complete Tn51098 harbouring OXA-244. Red triangles indicate absence of Type I fimbriae genes. As a reference, 28Eco12 was used (marked as reference). 
$\beta$-lactamase (ESBL) enzyme allele carried (i.e. CTX-M-27 and CTXM-14b, respectively) (Figure 1, Supplementary Table 2). CTX-M-27 is a point mutant derivative of CTX-M-14.

The ability of OXA-244 to hydrolyse imipenem and temocillin (a specificity of OXA-48 derivatives) is weaker than that of OXA48. Indeed, many of the isolates analysed here showed variable degrees of susceptibility/resistance to carbapenems and were often labelled as carbapenem-non-susceptible (or as susceptible, increased exposure according EUCAST 2019) when sent to the reference laboratories. Serial testing in the reference laboratories revealed variable results ranging from susceptible to resistant, according to EUCAST criteria in Minimal inhibitory concentration (MIC)-based breakpoint-testing. Using the Etest technique, the MICs for ertapenem, meropenem and imipenem ranged from 0.12$>32,0.04-1.5$ and $0.04-1 \mathrm{mg} / \mathrm{L}$, respectively. None of the strains was resistant to meropenem or imipenem (Supplementary Table 3, Supplementary Table 4). The low level (if any) of resistance to carbapenems observed for those OXA-244 producers may have been related to the low level of production of the OXA-244 enzyme be- cause the gene was present as a single copy on the chromosome in association with the low-level of carbapenem hydrolytic properties of this enzyme itself.

Co-resistances to other families of antibiotics (fluoroquinolones, tetracyclines, trimethoprim/sulfamethoxazole, aminoglycosides) were also highly similar within the strain collection (Supplementary Table 2, Supplementary Table 3, Supplementary Table 4). The bla $a_{\text {OXA-244 }}$ gene was chromosomally located in isolates from both lineages.

Clonal lineage I comprises isolates (21 of 31) that harbour the $b l a_{\mathrm{CTX}-\mathrm{M}-27}$ gene on an IncF plasmid. The $b l a_{\mathrm{OXA}-244}$-encoding mobile element of lineage I isolates does not resemble the classical Tn51098 or Tn6327 structure, as reported [8], but are deletion variants thereof (Figure 1, Supplementary Figure 2). Clonal lineage I isolates exhibited differences of $<129$ SNPs in their core genomes, regardless of whether the isolates were from Germany or Switzerland (Supplementary Figure 3) and almost exclusively of the serotype 086:H18 (Figure 1, Supplementary Table 11). They carried a Dr adhesin/invasion locus [9], but lacked Type I fimbriae

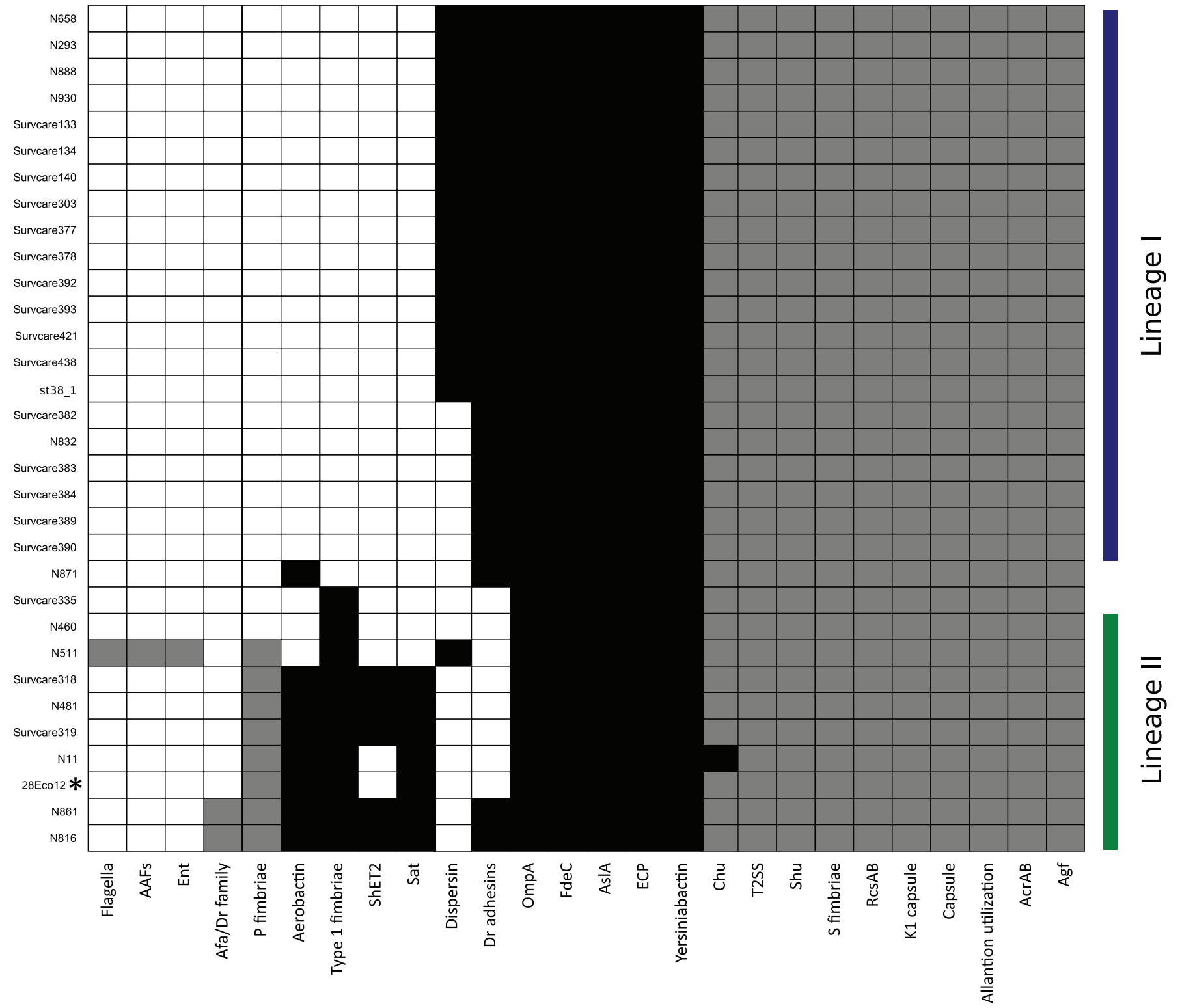

Figure 2. Virulence determinants of E. coli ST38 OXA-244 isolates. Complete operons are marked in black, incomplete operons in grey. The functions of the virulence determinants are indicated in Supplementary Table 5. The reference 28Eco12 is marked with an asterisk. 
(Figure 2). Isolates expressing the $\mathrm{Dr}$ adhesins are often associated with cystitis, pregnancy-associated pyelonephritis and chronic diarrhoea; thus, indicating that isolates of clonal lineage I may have virulence potential [9]. A representative isolate exhibited a diffuse adherent phenotype for adhesion to HeLa cells and was invasive (Supplementary Figure 4).

Clonal lineage II isolates (9 of 31) were slightly more heterogeneous but had a common chromosomal insertion site for the


eage II isolates resembled, in five of eight isolates, the previously described Tn51098 structure (Figure 1 and Supplementary Figure 2). They presented as several serotypes (0102:H6, ONT:H30 and $\mathrm{O} 2: \mathrm{H} 30$, Figure 1 ). In contrast to clonal lineage I, they encoded Type I fimbriae. Independent isolates of clonal lineage II from Germany and Switzerland were highly related (Figure 1, Supplementary Figure 3).

All ST38 isolates carried virulence and adaptive genes, including the iron uptake systems aerobactin and yersiniabactin as well as haeme-uptake and degradation systems (Figure 2). In contrast to classical uropathogenic E. coli (UPEC), they did not carry haemolysin genes.

It was noted that of the 31 isolates from both countries, 23 isolates were from female patients. This high proportion of female origin of the strains was evidenced among infections (mostly urinary tract infections), as expected, but also from colonisation. Two sets of OXA-244/CTX-M-27 isolates, SurvCARE382/383/384 (383 and 384 were twins) and SurvCARE392/393 represented mother/neonate combinations, while for one combination (SurvCARE 382/383/384/390) the neonates and one mother were present in the same room. The remaining isolates were almost exclusively obtained from elderly male patients (exceptions: SurvCARE377 and SurvCARE438). The fact that these lineages were isolated from patients of different ages and from different clinical specimens indicates an ability to colonise and infect.

A recent meta-analysis of 217 studies on commonly occurring extraintestinal E. coli (ExPEC) lineages worldwide in humans did not reveal a single isolate of ST38 prior to 2000 [10]. Nevertheless, a recent report documented an increase in ST38 isolates expressing OXA-244 throughout Germany since 2017 [11]. Also, OXA-244producing $E$. coli isolates have been identified from a number of countries within the EU, including The Netherlands, Spain, United Kingdom, and France [8]. Previous reports have indicated the presence of OXA-244 producers in the UK, Spain and France in strains belonging to the same ST38 type [12]. The large increase in OXA244 CTX-M-27/14b producing ST38 isolates in 2019 suggests an ongoing and emerging supranational clonal outbreak over a large area comprising parts of Germany, Switzerland and possibly many other European countries.

The clonal lineage II isolates encoding OXA-244/CTX-M-14b isolates harboured both ESBL and carbapenemase genes on the chromosome. This peculiar genetic situation may stabilise both genes in those $E$. coli strains, leading to a vertical transfer of this resistance trait. Therefore, any $\beta$-lactam used for treating $E$. coli infections (penicillin, penicillin/ $\beta$-lactamase inhibitor, cephalosporin, carbapenem) may select those OXA-244 producers, whereas OXA-244, like other OXA-48-like enzymes, does not hydrolyse cephalosporins. This genetic background may be of the utmost importance for explaining a further spread of communityacquired OXA-244 producers.

It has previously been suggested that DAEC isolates act as 'silent pathogens' that are well-controlled in healthy individuals but express pathogenic potential in susceptible hosts [9]. Thus, the combination of under-detection of multidrug-resistant OXA-244 producing bacteria due to their low level of resistance to carbapenems (if any) and their properties as conditional pathobionts are worrying, and likely to favour their silent spread worldwide.

\section{Acknowledgements}

We thank the participating clinics for contribution of isolates to SurvCARE.

We thank the following Swiss colleagues for providing the clinical strains within the NARA network: C. Ottinger (Aarau); A. Egli (Basel); K. Burren, S. Thiermann and R. Troesch (Bern); V. Deggim, D. Bandeira and S. Pfister (Fribourg); L. Bandollier, N. Burri, M. Brandenberger, P. Friderich, S. Imhof, E. Mazenauer, I. Mitrovic and B. Suter Buser (Luzern); C. Andreutti, D. Blanc, M. Dessauges, V. Dilorenzo, G. Prod'Hom, G. Greub, C. Payen and V. Slutter (Lausanne); S. Emoney, M. Eyer and L. Tissieres (Sion); and G. Eich (Zürich).

We thank Julie Kessler, Anthony Demords, Christina Gerstmann, Sylvia Krämer, Martina Hudel, Julia Baldauf and Gudrun BettgeWeller for excellent technical assistance.

\section{Declarations}

Funding: This work was carried out by grants from the Bundesministerium fuer Bildung und Forschung (BMBF, Germany) within the German Center for Infection research (DZIF/grant number, 8032808818 , and 8032808820 to $\mathrm{TC} / \mathrm{CI}$ ) and the University of Fribourg by the Swiss National Reference center for Emerging Antibiotic Resistance and by the Swiss National Science Foundation (project FNS-31003A_163432). Support was also obtained by the Hessian State Ministry for Social Affairs and Integration (HMSI) within the project SurvCARE Hessen and the Hessian Ministry of Higher Education, Research and Arts within the project HuKKH (Hessisches universitaeres Kompetenzzentrum Krankenhaushygiene). The funders of the study played no role in study design, data collection, data analysis, data interpretation or writing the report.

\section{Competing Interests: None.}

Ethical Approval: Not applicable.

Author contributions: LF, PN and TC designed the study. PN, CI, $\mathrm{AH}, \mathrm{PH}$ and TC provided data and isolates. LF, PN, CI, YY, JF, PH and TC gathered and analysed the data. LF, PN and TC wrote the manuscript, which all authors approved.

\section{Supplementary materials}

Supplementary material associated with this article can be found, in the online version, at doi:10.1016/j.ijantimicag.2020. 106157.

\section{References}

[1] Nordmann P, Poirel L. Epidemiology and diagnostics of carbapenem resistance in Gram-negative bacteria. Clin Infect Dis 2019;69:S521-8.

[2] The European Committee on Antimicrobial Susceptibility Testing (EUCAST). Breakpoints tables for interpretation of MICs and zone diameters. Available at: https://www.eucast.org/fileadmin/src/media/PDFs/EUCAST_files/Breakpoint_ tables/v_10.0_Breakpoint_Tables.pdf.

[3] Masseron A, Poirel L, Falgenhauer L, Imirzalioglu C, Kessler J, Chakraborty T, et al. Ongoing dissemination of OXA-244 carbapenemase-producing Escherichia coli in Switzerland and their detection. Diagn Microbiol Infect Dis 2020;97:115059.

[4] Schwengers O, Hoek A, Fritzenwanker M, Falgenhauer L, Hain T, Chakraborty C, et al. $\mathrm{ASA}^{3} \mathrm{P}$ : An automatic and scalable pipeline for the assembly, annotation and higher level analysis of closely related bacterial isolates. PLoS Comput Biol 2020;16:e1007134.

[5] Treangen TJ, Ondov BD, Koren S, Phillippy AM. The Harvest suite for rapid core-genome alignment and visualization of thousands of intraspecific microbial genomes. Genome Biol 2014;15:524.

[6] Croucher NJ, Page AJ, Connor TR, Delaney AJ, Keane JA, Bentley SD, et al. Rapid phylogenetic analysis of large samples of recombinant bacterial whole genome sequences using Gubbins. Nucleic Acids Res 2015;43:e15. 
7] Clermont O, Bonacorsi S, Bingen E. Rapid and Simple Determination of the Escherichia coli Phylogenetic Group. Appl Environ Microbiol 2000;66:4555-8.

[8] Pitout JDD, Peirano G, Kock MM, Strydom KA, Matsumura Y. The Global Ascendency of OXA-48-Type Carbapenemases. Clin Microbiol Rev 2020;33 e00102-19.

[9] Servin AL. Pathogenesis of human diffusely adhering Escherichia coli expressing Afa/Dr adhesins (Afa/Dr DAEC): current insights and future challenges. Clin Microbiol Rev 2014;27:823-69.

[10] Manges AR, Geum HM, Guo A, Edens TJ, Fibke CD, Pitout JDD. Global Extraintestinal Pathogenic Escherichia coli (ExPEC) Lineages. Clin Microbiol Rev 2019;32 e00135-18.
[11] Kremer K, Kramer R, Neumann B, Haller S, Pfennigwerth N, Werner G, et al. Rapid spread of OXA-244-producing Escherichia coli ST38 in Germany: insights from an integrated molecular surveillance approach; 2017 to January 2020. Euro Surveill 2020;25:2000923.

[12] Hoyos-Mallecot Y, Naas T, Bonnin RA, Patino R, Glaser P, Fortineau N, et al. OXA-244-producing Escherichia coli isolates, a challenge for clinical microbiology laboratories. Antimicrob Agents Chemother 2017;61 e00818-17. 